\title{
Top quark pair-production cross-section measurements with the ATLAS detector
}

\author{
Pavol Bartoš $^{*}$ on behalf of the ATLAS collaboration ${ }^{\dagger}$ \\ Comenius University \\ E-mail: pavol.bartosecern.ch
}

\begin{abstract}
Measurements of the inclusive and differential top-quark pair cross sections in proton-proton collisions at both 8 and $13 \mathrm{TeV}$ with the ATLAS detector at the Large Hadron Collider are presented. The inclusive measurements reach high precision and are compared to the best available theoretical calculations. Differential measurements of the kinematic properties of the top quark production are also discussed. These measurements, including results using boosted top quarks, probe our understanding of top-quark pair production in the $\mathrm{TeV}$ regime. The results, unfolded to particle and parton level, are compared to predictions of Monte Carlo generators implementing NLO matrix elements matched with parton showers and NNLO QCD theory calculations.
\end{abstract}

XXVI International Workshop on Deep-Inelastic Scattering and Related Subjects (DIS2018) 16-20 April 2018

Kobe, Japan

\footnotetext{
* Speaker.

${ }^{\dagger}$ Work supported by the Ministry of Education, Science, Research and Sport of the Slovak Republic.
} 


\section{Introduction}

The top quark is the heaviest elementary particle of the Standard model (SM). At the Large Hadron Collider [1], the top quark is mainly produced in quark-antiquark pairs $(t \bar{t})$ through gluon fusion or quark-antiquark annihilation. Due to a very short life-time, top quark decays before hadronisation. According to the SM, top quark decays into $W$ boson and $b$ quark in almost $100 \%$ of the time. For the $t \bar{t}$ production, one can distinguish three decay channels: all-hadronic, lepton+jets, and dilepton channel, which contains 0,1 , and 2 leptonically decaying $W$ bosons, respectively. Usually, only electron and muons are considered as leptons.

Precise measurements of $t \bar{t}$ production cross-section $\left(\sigma_{t \bar{t}}\right)$ allow us to improve Monte Carlo modelling, constrain parton distribution functions (PDF). As the top quark is produced at large energy scale characterised by a low value of coupling constant $\left(\alpha_{s} \approx 0.1\right)$, it is excellent perturbative object for testing quantum chromodynamics (QCD). Moreover we can set limits on new physics scenarios beyond the Standard model. This paper presents results of $\sigma_{t \bar{t}}$ measurements in all three decay channels, obtained by the ATLAS Collaboration [2].

\section{Inclusive $t \bar{t}$ cross-section}

Using the data of integrated luminosity of $3.2 \mathrm{fb}^{-1}$ collected in proton-proton collisions at $\sqrt{s}=13 \mathrm{TeV}$, the most precise measurement of the inclusive $\sigma_{t \bar{t}}$ is obtained from the dilepton decay channel [3]. In this measurement, events with opposite sign leptons are selected. To suppress the $Z+$ jets background, only events with one electron and one muon were used. The measured crosssection of $\sigma_{t \bar{t}}=818 \pm 8$ (stat.) \pm 27 (syst.) \pm 19 (lumi) \pm 12 (beam) pb has precision of $4.4 \%$ which is better than precision of the theoretical prediction (5.5\%) [4].

The summary of the ATLAS measurements of the inclusive $\sigma_{t \bar{t}}$ is shown in Fig. 1. However, there are new measurements, which are not shown on the plot, or which supersede the shown results. The results are in good agreement with the next-to-next-to-leading-order (NNLO) QCD calculation complemented with next-to-next-to-leading-logarithmic resummation [4].

In the lepton+jets channel, new measurement of inclusive $\sigma_{t \bar{t}}$ at $\sqrt{s}=8 \mathrm{TeV}$ using the data sample of $20.2 \mathrm{fb}^{-1}$ [5] supersedes results from [6]. The selected events are required to contain one electron or muon, at least four small-R jets ${ }^{1}$, and a missing transverse energy (due to presence of a neutrino). At least one of the small- $R$ jets is required to be tagged as jet initiated by a $b$ quark ( $b$-jet). The selected data is divided into three signal regions depending on number of small$R$ jets and number of $b$-jets. A discriminating variable is used to suppress the background. The final result is obtained from binned maximum-likelihood fit performed simultaneously in all three signal regions. The fiducial cross-section ${ }^{2}$ of $\sigma_{t \bar{t}}^{\text {fid }}=48.8 \pm 0.1$ (stat.) \pm 2.0 (syst.) \pm 0.9 (lumi) pb is measured with relative uncertainty of $4.5 \%$. The inclusive cross-section of $\sigma_{t \bar{t}}^{\text {incl }}=248.3 \pm$ 0.7 (stat.) \pm 13.4 (syst.) \pm 4.7 (lumi) pb with relative uncertainty of $5.7 \%$ is in good agreement with theory prediction of $\sigma_{t \bar{t}}^{\text {theo }}=253_{-15}^{+13} \mathrm{pb}$. The largest contribution to the systematic uncertainty comes from signal Monte Carlo modelling and uncertainty on PDFs.

\footnotetext{
${ }^{1}$ Jets are reconstructed with anti- $k_{t}$ algorithm, where $R$ is a radius parameter. For small- $R$ jets, $R=0.4$.

${ }^{2}$ The fiducial phase-space is defined by selecting events with exactly one electron or muon and at least three particlelevel jets.
} 


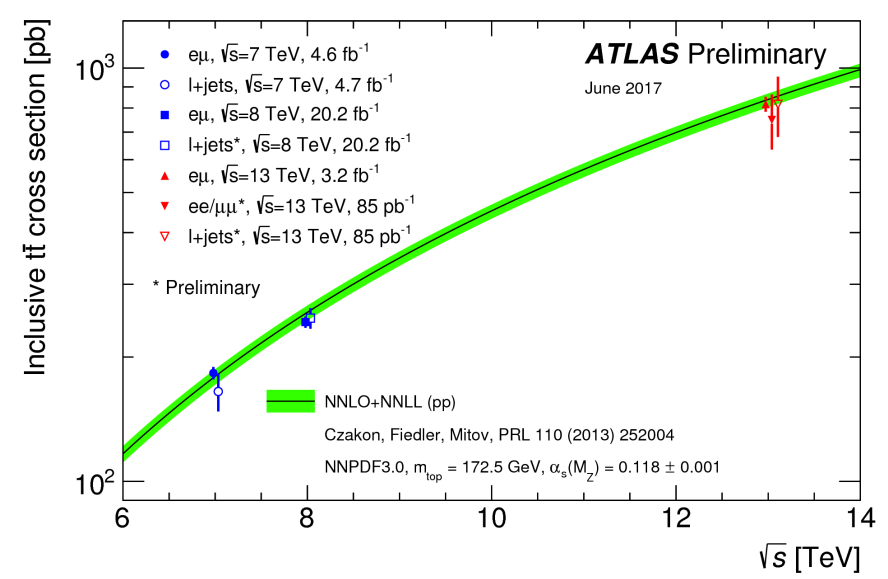

Figure 1: Summary of ATLAS measurements of the top-pair production cross-section as a function of the centre-of-mass energy compared to the NNLO QCD calculation complemented with NNLL resummation. The theory band represents uncertainties due to renormalisation and factorisation scale, parton density functions and the strong coupling [8].

In addition, the inclusive $\sigma_{t \bar{t}}$ at $\sqrt{s}=8 \mathrm{TeV}$ using the data sample of $20.2 \mathrm{fb}^{-1}$ is measured in $\tau+$ jets events with hadronically decaying $\tau$ lepton [7]. The events with one $\tau$ lepton, at least two small- $R$ jets, and missing transverse energy are selected. Furthermore, at least two $b$-jets are required. The hadronically decaying $\tau$ can contain one or three charged particles in the $\tau$-jet, while non-zero number of neutral pions is allowed. In case of three charged particles inside the $\tau$-jet, the sum of particles' charges has to be one. A boosted decision tree is used to distinguish $\tau$-jets from the jets initiated by a quark or gluon. The measured cross-section of $\sigma_{t \bar{t}}^{\text {incl }}=239 \pm$ 4 (stat.) \pm 28 (syst.) \pm 5 (lumi) pb has relative uncertainty of $12 \%$. The main sources of systematic uncertainty are uncertainties in radiation, jet energy scale and $b$-tagging efficiency. Using this decay channel, one can investigate coupling of the $3^{\text {rd }}$ generation fermions in a single process. Deviation in $B R\left(t \rightarrow \tau V_{\tau} b\right)$ would indicate a non-SM physics. The observed (expected) upper limit at $95 \%$ confidence level on the visible cross section of any non-SM process is $22\left(22_{-1}^{+2}\right) \mathrm{fb}$ [7].

The experimental precision of the total $t \bar{t}$ production cross section, $\sigma_{t \bar{t}}^{\text {incl }}$, has reached the fewpercent level, while the precision of the $Z$ boson production cross section measured in $Z \rightarrow \ell^{+} \ell^{-}$ decay channel within the fiducial region defined by the detector acceptance, $\sigma_{Z}^{\text {fid }}$, is at sub-percent level. The experimental precision is complemented by an accurate determination of the $p p$ collision luminosity, which is at level of $\approx 2 \%$. By defining ratio of $\sigma_{t \bar{t}}^{\text {incl }} / \sigma_{Z}^{\text {fid }}$ the luminosity and some of experimental uncertainties are cancelled. The ratio has a significant sensitivity to the gluon-toquark PDF ratio. The ratio is extracted from the previously measured cross-sections, except the $\sigma_{Z}^{\text {fid }}$ measurement at $\sqrt{s}=13 \mathrm{TeV}$, which has been measured for the first time [9]. The fiducial phase-space for $Z$ boson production is defined by selection criteria applied on lepton transverse momenta $\left(p_{\mathrm{T}}^{\ell}>25 \mathrm{GeV}\right)$, its pseudorapidity $\left(\left|\eta^{\ell}\right|<2.5\right)$, and by requiring dilepton invariant mass to be in $Z$ boson mass window $\left(66<m_{\ell \ell}<116 \mathrm{GeV}\right)$. Figure 2 shows the ratio of $\sigma_{t \bar{t}}^{\text {incl }} / \sigma_{Z}^{\text {fid }}$ for $\sqrt{s}=13 \mathrm{TeV}$ compared to predictions based on different PDF sets. The data agrees the best with ATLAS-epWZ12 PDF set and disfavours ABM12 PDF set [9]. The relative uncertainty of the 
measured ratio is $3.6 \%$.

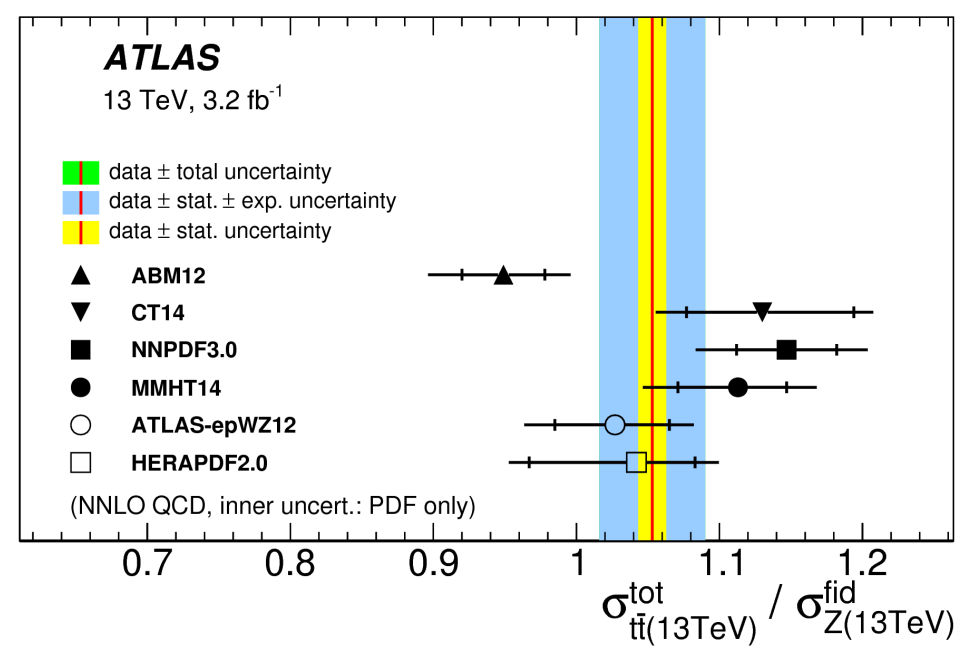

Figure 2: The ratio of $\sigma_{t \bar{t}}^{\text {incl }} / \sigma_{Z}^{\text {fid }}$ for $\sqrt{s}=13 \mathrm{TeV}$ compared to predictions based on different PDF sets. The inner shaded band corresponds to the statistical uncertainty, the middle band to the statistical and experimental systematic uncertainties added in quadrature, while the outer band shows the total uncertainty, including the luminosity uncertainty. The theory predictions are given with the corresponding PDF uncertainties shown as inner bars while the outer bars include all other uncertainties added in quadrature [9].

\section{Differential țt cross-section}

The differential $t \bar{t}$ cross-section is measured in all three decay channels while the standard event selection is used $[10,11,12]$. In the lepton+jet channel two topologies are considered: resolved one, where it is possible to identify all decay products separately; and boosted one, where the decay products of the hadronically decaying top quark are colimated into one large jet reconstructed with radius parameter of $R=1.0$ (large- $R$ jet). In the all hadronic channel only events with both top-quarks being boosted are considered ( $\geq 2$ large- $R$ top-tagged jets). In all three decay channels the iterative Bayesian unfolding is used to correct for detector effects.

The differential cross-section is measured as a function of several variables:

- $\mathrm{p}_{\mathrm{T}}$ of top quark, invariant mass of the $\overline{\mathrm{t}} \mathrm{system}, m^{\mathrm{t} \overline{\mathrm{t}}}$, which are sensitive to the modelling of higher order corrections in QCD.

- rapidity of top quark, $\mathrm{y}^{\mathrm{t}}$, and $\mathrm{t} \overline{\mathrm{t}}$ system, $\mathrm{y}^{\mathrm{t}}$, which are sensitive to the PDFs.

- $\mathrm{p}_{\mathrm{T}}$ of $\mathrm{tt}$ system, $\mathrm{p}_{\mathrm{T}}{ }^{\mathrm{t}}{ }^{\mathrm{t}}$, which is sensitive to the amount of gluon radiation in the event, useful for the tuning of Monte Carlo generators.

The dependencies on variables listed above are measured in all three decay channels. Some other dependencies can be found in references. If it is not mentioned otherwise, there is an agreement between measured data and predictions.

Figure 3 shows relative differential cross-sections as a function of the $p_{\mathrm{T}}$ of the hadronic top quark in fiducial phase-space in the resolved topology. A tension between data and the most of the 
predictions can be seen. The electro-weak corrections are not included in the predictions, but the corrections would not be large enough to remove discrepancy for $p_{\mathrm{T}} \sim 1 \mathrm{TeV}$ [10]. Further studies of top-quark $p_{\mathrm{T}}$ dependence can be found in A. Hasib's contribution or in [13].

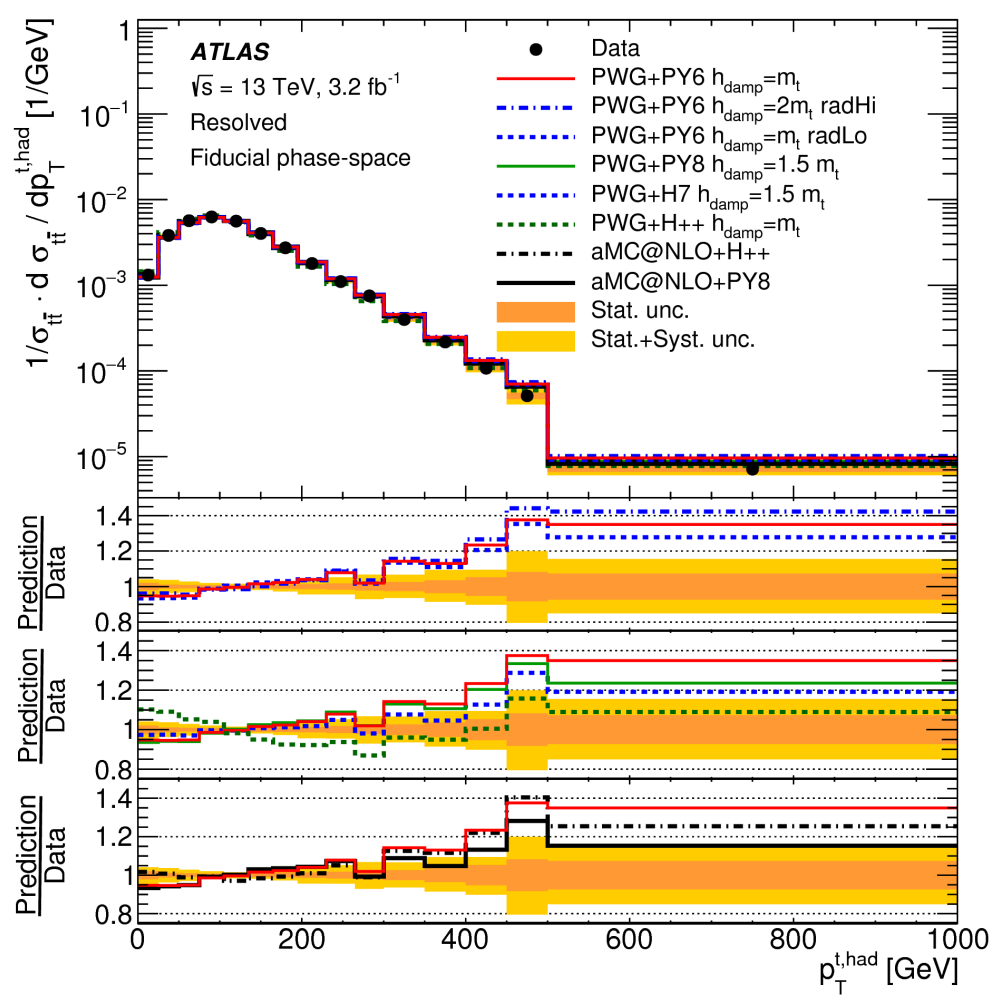

Figure 3: The relative differential cross-sections as a function of the $p_{\mathrm{T}}$ of the hadronic top quark in fiducial phase-space in the resolved topology. The yellow bands indicate the total uncertainty of the data in each bin. The lower three panels show the ratio of the predictions to the data [10].

For dependencies on $m^{\mathrm{t} \overline{\mathrm{t}}}$ and $y^{\mathrm{t} \bar{t}}$ the data reasonably agree with predictions except for HERWIG++ samples in lepton+jets and dilepton channels. In all-hadronic channel there is no comparison with HERWIG++ as HERWIG7 is used instead. The $t \bar{t}$ cross-section as a function of $y^{\mathrm{t}}$ obtained from data is a bit broader than predictions in all-hadronic channel. The dependence on $p_{T}^{\mathrm{t}} \overline{\mathrm{t}}$ shows a disagreement between data and predictions at high $p_{\mathrm{T}}^{\mathrm{t} \mathrm{t}}$ values in lepton+jets channel, while in all-hadronic channel there are predictions with both, softer and also with harder spectrum.

In all-hadronic channel a cross-section in fiducial phase-space defined by transverse momenta of top quarks $\left(p_{\mathrm{T}}^{\mathrm{t}, 1}>500 \mathrm{GeV}, p_{\mathrm{T}}^{\mathrm{t}, 2}>350 \mathrm{GeV}\right.$ ) is measured to be $\sigma_{t \bar{t}}^{\mathrm{fid}}=292 \pm 7$ (stat. $) \pm$ 76(syst.) fb, what is in agreement with POWHEG+PYTHIA8 predictions [12].

\section{Conclusions}

Results on top-quark pair-production from the ATLAS experiment at the LHC have been presented. The inclusive cross-section reaches precision at the level of the precision of theory predictions or better. The measurements of the differential cross-section explore large phase space. The variables used in the measurements are sensitive to QCD modelling and tuning of Monte Carlo 
generators. The measured data are comparable with theory predictions within uncertainties. However, there is some tension between measurement and the NLO predictions for some quantities (e.g. transverse momenta of top-quark).

\section{Acknowledgments}

It is a pleasure to thank the ATLAS collaborators for their well-done work, the conveners for their help and the organizers of the DIS 2018 for a very interesting workshop.

\section{References}

[1] L. Evans and P. Bryant (editors), LHC Machine, JINST 3 (2008) S08001.

[2] ATLAS Collaboration, The ATLAS Experiment at the CERN Large Hadron Collider, JINST 3 (2008) S08003.

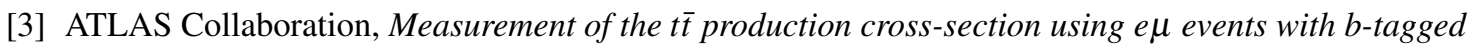
jets in pp collisions at $\sqrt{s}=13 \mathrm{TeV}$ with the ATLAS detector, Phys. Lett. $\mathbf{B 7 6 1}$ (2016) 136 [hep-ex/1606.02699].

[4] M. Czakon, P. Fiedler, A. Mitov, Total Top-Quark Pair-Production Cross Section at Hadron Colliders Through $O\left(\alpha_{S}^{4}\right), P R L 110$ (2013) 252004, [hep-ph/1303.6254].

[5] ATLAS Collaboration, Measurement of the inclusive and fiducial t $\bar{t}$ production cross-sections in the lepton+jets channel in pp collisions at $\sqrt{s}=8$ TeV with the ATLAS detector, Eur. Phys. J. C78 (2018) 487, [hep-ex/1712.06857].

[6] ATLAS Collaboration, Measurement of the top pair production cross-section in $8 \mathrm{TeV}$ proton-proton collisions using kinematic information in the lepton+jets final state with ATLAS, Phys. Rev. D91 (2015) 112013, [hep-ex/1504.04251].

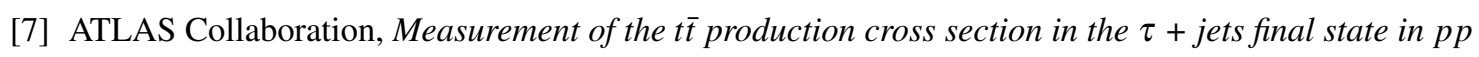
collisions at $\sqrt{s}=8$ TeV using the ATLAS detector, Phys. Rev. D95 (2017) 072003,

[hep-ex/1702.08839].

[8] ATLAS Collaboration, Summary plots from the ATLAS Top physics group, https://atlas.web.cern.ch/Atlas/GROUPS/PHYSICS/CombinedSummaryPlots/TOP/

[9] ATLAS Collaboration, Measurements of top-quark pair to Z-boson cross-section ratios at $\sqrt{s}=13,8,7 \mathrm{TeV}$ with the ATLAS detector, JHEP 02 (2017) 117, [hep-ex/1612.03636].

[10] ATLAS Collaboration, Measurements of top-quark pair differential cross-sections in the lepton+jets channel in pp collisions at $\sqrt{s}=13 \mathrm{TeV}$ using the ATLAS detector, JHEP 11 (2017) 191, [hep-ex/1708.00727].

[11] ATLAS Collaboration, Measurement of lepton differential distributions and the top quark mass in $t \bar{t}$ production in pp collisions at $\sqrt{s}=8 \mathrm{TeV}$ with the ATLAS detector, Eur. Phys. J. C77 (2017) 804, [hep-ex/1709.09407].

[12] ATLAS Collaboration, Measurements of $t \bar{t}$ differential cross-sections of highly boosted top quarks decaying to all-hadronic final states in pp collisions at $\sqrt{s}=13 \mathrm{TeV}$ using the ATLAS detector, Phys. Rev. D98 (2018) 012003, [hep-ex/1801. 02052].

[13] ATLAS Collaboration, Measurements of differential cross sections of top quark pair production in association with jets in pp collisions at $\sqrt{s}=13 \mathrm{TeV}$ using the ATLAS detector, CERN-EP-2017-227, https://cds.cern.ch/record/2304924, Submitted to JHEP, [hep-ex/1802.06572]. 